\title{
A GLOBAL VERSION OF GEHRING LEMMA IN ORLICZ SPACES ON SPACES OF HOMOGENEOUS TYPE
}

\author{
MARCELINA MOCANU
}

Abstract. We extend a global version of Gehring lemma in Orlicz spaces, proved by T. Iwaniec, from the Euclidean case to the setting of spaces of homogeneous type.

Mathematics subject classification (2010): 42B25, 46E30.

Keywords and phrases: Gehring lemma, space of homogeneous type, Calderón-Zygmund decomposition, Orlicz space.

\section{REFERENCES}

[1] A. BJÖRn And J. BJÖRn, Nonlinear Potential Theory on Metric Spaces, EMS Tracts in Mathematics 17, 2011, $403 \mathrm{pp}$

[2] A. Cianchi, Some results in the theory of Orlicz spaces and applications to variational problems, Nonlinear Analysis, Function Spaces and Applications 6 (1999), 50-92.

[3] A. CIANCHI AND N. FusCO, Gradient regularity for minimizers under general growth conditions, J. Reine Angew. Math., 507 (1999), 15-36.

[4] R. Coifman AND G. Weiss, Analyse Harmonique Non-commutative sur Certaines Espaces Homogènes: Etude de Certaines Integrals Singulieres, Lecture Notes in Mathematics, book 242, Springer, 1971, $162 \mathrm{pp}$.

[5] B. Franchi AND F. SERRA CASSANO, Gehring's lemma for metrics and higher integrability of the gradient for minimizers of noncoercive variational functionals, Studia Math. 120, 1 (1996), 1-22.

[6] F. W. GEHRING, The $L^{p}$ - integrability of the partial derivatives of a quasiconformal mapping, Acta Math. 130 (1973), 265-277.

[7] U. GianaZZA, The $L^{p}$ integrability on homogeneous spaces, Ist. Lombardo Accad. Sci. Lett. Rend. A, 126 (1992), 83-92.

[8] L. GReCo AND G. ZeCCA, A version of Gehring lemma in Orlicz spaces, Rendiconti Lincei. Matematica e Applicazioni, vol. 23, 1 (2012), 29-50.

[9] L. Grafakos, L. Liu, D. Maldonado and D. Yang, Multilinear analysis in metric spaces, Dissertationes Math. 497 (2014), 121 pages, doi:10.4064/dm497-0-1

[10] P. Hajeasz and P. Koskela, Sobolev met Poincaré, Mem. Amer. Math. Soc. vol. 145, no. 688 (2000), 103 pp.

[11] J. Kinnunen, Higher integrability with weights, Ann. Acad. Sci. Fenn., Ser. A.I. Math., 19 (1994), $355-366$.

[12] T. Iwaniec, The Gehring lemma, in Quasiconformal Mappings and Analysis, Springer, 1998, pp. 181-204.

[13] T. Iwaniec And G. Martin, Geometric Function Theory and Nonlinear Analysis, Oxford Science Publications, Clarendon Press, 2001, 552 pp.

[14] O.E. MaASAlo, Self-improving phenomena in the calculus of variations on metric spaces, Ph.D. thesis, Helsinki University of Technology, 2008.

[15] J. Martin And M. Milman, Gehring's lemma for nondoubling measures, Michigan Math. J. 47, 3 (2000), 559-573.

[16] M. MastyŁo And M. Milman, A new approach to Gehring's lemma, Indiana Univ. Math. J. 49, 2 (2000), 655-680.

[17] M. Milman, A note on Gehring lemma, Ann. Acad. Sci. Fenn., Ser. A.I. Math., 21 (1996), 389-398. 
[18] H. Tuominen, Orlicz-Sobolev spaces on metric measure spaces, Ann. Acad. Sci. Fenn. Math. Dissertationes 135 (2004), 279 pp.

[19] A. Zatorska-Goldstein, Very weak solutions of nonlinear elliptic equations, Ann. Acad. Sci. Fenn., Ser. A.I. Math., 30 (2005), 407-436. 